\title{
Burden and outcome of neonatal surgical conditions in Nigeria: A countrywide multicenter cohort study
}

Ekwunife Okechukwu $\mathrm{H}^{,{ }^{*}}$ Ameh Emmanuel $\mathrm{A},{ }^{2}$ AbdurRahman Lukman $\mathrm{O},{ }^{3}$ Ademuyiwa Adesoji, ${ }^{4}$ Akpanudo Emem, ${ }^{5}$ Alakaloko Felix, ${ }^{4}$ Bwala Kefas, ${ }^{6}$ Egbuchulem Ifeanyichukwu $K,{ }^{7}$ Ekenze Sebastian $0,{ }^{8}$ Ezomike Uchechukwu $0,{ }^{8}$ Faboya Omolara $M,{ }^{9}$ Ladipo-Ajayi Oluwaseun, ${ }^{4}$ Lawal Taiwo $A,{ }^{7}$ Lukong Christopher, ${ }^{10}$ Modekwe Victor I, ${ }^{1}$ Nasir Abdulrasheed A, ${ }^{3}$ Nwokoro Chigbundu $C,{ }^{11}$ Okafor Dave, ${ }^{12}$ Okoro Philemon E, ${ }^{13}$ Olori Samson, ${ }^{14}$ Orji Emmanuel, ${ }^{12}$ Seyi-Olajide Justina $0,{ }^{4}$ Sholadoye Tunde ${ }^{15}{ }^{15}$ Sowande Oludayo, ${ }^{16}$ Taiwo Jones $0,{ }^{17}$ Tanimola Adebayo, ${ }^{16}$ Ugwu Jideofor $0,{ }^{1}$ Williams Omolara $\mathrm{M},{ }^{9}$

Correspondence*: Okechukwu H Ekwunife, Surgery Department, Nnamdi Azikiwe University / Nnamdi Azikiwe University Teaching Hospital Nnewi, Anambra State Nigeria. E-mail: oh.ekwunife@unizik.edu.ng

\section{KEYWORDS \\ Neonatal surgery, Complications, \\ Mortality, \\ Predictors, \\ Quality improvement}

\begin{abstract}
Background: Despite a decreasing global neonatal mortality, the rate in sub-Saharan Africa is still high. The contribution and the burden of surgical illness to this high mortality rate have not been fully ascertained. This study is performed to determine the overall and disease-specific mortality and morbidity rates following neonatal surgeries; and the pre, intra, and post-operative factors affecting these outcomes.
\end{abstract}

\begin{abstract}
Methods: This was a prospective observational cohort study; a country-wide, multi-center observational study of neonatal surgeries in 17 tertiary hospitals in Nigeria. The participants were 304 neonates that had surgery within 28 days of life. The primary outcome measure was 30-day postoperative mortality and the secondary outcome measure was 30-day postoperative complication rates.
\end{abstract}

Results: There were 200 (65.8\%) boys and 104 (34.2\%) girls, aged 1-28 days (mean of $12.1 \pm$ 10.1 days) and $99(31.6 \%)$ were preterm. Sepsis was the most frequent major postoperative complication occurring in $97(32 \%)$ neonates. Others were surgical site infection $(88,29.2 \%)$ and malnutrition $(76,25.2 \%)$. Mortality occurred in 81 (26.6\%) neonates. Case-specific mortalities were: gastroschisis $(14,58.3 \%)$, esophageal atresia $(13,56.5 \%)$ and intestinal atresia $(25,37.2 \%)$. Complications significantly correlated with 30 -day mortality $(\mathrm{p}<0.05)$. The major risk predictors of mortality were apnea $(\mathrm{OR}=10.8)$, severe malnutrition $(\mathrm{OR}=6.9)$, sepsis $(\mathrm{OR}=7$. I), deep surgical site infection $(\mathrm{OR}=3.5)$, and re-operation $(\mathrm{OR}=2.9)$.

Conclusion: Neonatal surgical mortality is high at $26.2 \%$. Significant mortality risk factors include prematurity, apnea, malnutrition, and sepsis.

\section{INTRODUCTION}

Sustainable Development Goal (SDG) number 3 sets out among other things to decrease neonatal mortality to as low as 12 per 1000 live births by 2030. [1] It is estimated that at the current trend, more than 60 countries will miss the SDG target by 2030. [2] Most sub-Sahara African countries belong to this category. There is thus an urgent need to improve the accessibility and quality of surgical care.

Despite a decreasing global Neonatal Mortality Rate (NMR), there is still a huge disparity in sub-Saharan Africa with a slower decline when compared to the global statistics. Congenital anomalies which form the bulk of most neonatal surgical cases globally constitute $10.6 \%$ of NMR. Congenital anomaly is currently the 5 th leading cause of neonatal mortality worldwide and is likely to continue rising in profile. [4] Low- and
Middle-Income Countries (LMICs) may have high mortality from surgical deaths given the high incidence of congenital anomalies and the prevalent high underreporting rate. [4]

With 359,081 neonates dying in Nigeria in 2019, NMR in Nigeria is among the highest globally at 47.7 per 1000 population. [5]

Nigeria as classified by the World Bank based on Gross National Income is a lower-middle-income country. The practice of neonatal surgery in Nigeria as in most LMICs is still faced with multiple challenges. Although some isolated improvements have been made in some centers, recent outcome reports still have mortality rates of up to $50 \%$, with a range across institutions as $12 \%$ to $62 \%$. [6-10] Not much has changed regarding the challenges militating against good outcomes. [11] The contribution of surgical ill- 
ness to this high mortality rate has not been fully ascertained.

There is thus a need to determine the actual burden and outcome of neonatal surgical conditions in Nigeria to plan and implement strategies to improve the outcomes. The objectives of this study are first, to determine the overall and disease-specific mortality and morbidity rates following neonatal surgery in $\mathrm{Ni}$ geria. Secondly, to study the pre-, intra-, and postoperative factors affecting the outcome of neonatal surgery. These will provide a baseline for planning the implementation of mitigation strategies.

\section{METHODS}

\section{Study Design and Setting}

This was a collaborative prospective cohort study conducted from May 2018 -December 2019 involving twenty-eight investigators from 17 tertiary hospitals.

\section{Data Collection Tool}

The study tool was adapted from the American College of Surgeons National Surgical Quality Improvement Programme Paediatric (ACS NSQIP-P). [12]

Information from enrolled participants on demography, presentation, diagnosis, treatment, outcome, and factors affecting outcome was collated using a customized web-based data capture tool.

Patients were monitored and data was collected up to 30 days post-surgery.

Collaborators were trained virtually on the study protocol and study progress was regularly monitored by the principal investigator.

\section{Inclusion and Exclusion Criteria}

Consecutive neonates from the selected centers aged 0-28 days at the time of presentation, who had a surgical disease and surgical intervention in the neonatal period were enrolled in the study and were followed up for 30 days after surgery.

Circumcision, orthopedic, plastic, neurosurgical, or cardiac surgeries were excluded.

\section{Sample Size and Sampling}

Most pediatric centers in Nigeria perform on average, about 50 general neonatal surgical operations in a year. [6,9,13] Although 800 subjects were the total predicted population, the minimum sample size at $95 \%$ confidence level and 5\% margin of error was 260 . However, we enrolled 304 patients for the study. [14]

Seventeen centers were selected by purposive sampling method based on the frequency of neonatal surgeries and evenly distributed across the six regions. Each included hospital has at least one trained pediatric surgeon.

\section{Data Capture Monitoring and Validation}

A two-week pilot study was conducted to test and validate the study tool and the customized web-based data capture before the commencement of the study and necessary adjustments made. Monthly interactions with study centers via phone calls and media messaging were used to monitor compliance and ensure data integrity.

\section{Ethics}

Ethical approval for the study was obtained from the Health Research and Ethics Committee (HREC) of Nnamdi Azikiwe University Teaching Hospital (NAUTH/CS/66/VOL 10/208/2017/106).

Informed consent was obtained from the participants. Being an observational study with little or no influence on the choice of treatment, no parent declined consent or withdrew from the study.

\section{Data Analysis}

Data were analyzed using STATA ${ }$ Statistics/Data Analysis version 16.0 (StataCorp LLC Texas USA). Tables and charts were used to present the data. A Chi-square test was done to test associations between variables. Logistic regression analysis was done to assess the risks of mortality. The level of statistical significance was set at $\mathrm{p} \leq 0.05$.

\section{Outcome Measures}

The primary outcome measure was 30-day postoperative mortality. Secondary outcome measures were 30day postoperative complication rates and adverse perioperative events.

\section{RESULTS}

\section{Demographics}

There were 104 (34.2\%) girls and 200 (65.8\%) boys aged 0-28 days (mean 12.1 \pm 10.1 days). While $99(31.6 \%)$ were born preterm; $18(5.0 \%)$ were extremely preterm (Table 1$)$. Only $72(23.7 \%)$ of the infants lived within $30 \mathrm{~km}$ distance from the hospital, $172(56.6 \%)$ had to travel more than $50 \mathrm{~km}$, and $18(5.9 \%)$ more than $100 \mathrm{~km}$ to access care. The most common place of delivery of the neonates was in private non-governmental hospitals (92, 30.3\%) followed by unsupervised delivery at home in 61 (20.0\%). One hundred and forty-five (47.2\%) infants arrived at the hospital with their caregivers using public transportation while $11(3.6 \%)$ arrived with an ambulance service. Surgical neonates were managed mostly in Special Care Baby Units or High Dependency Units.

\section{Time of presentation}

The duration of presentation from the onset of symptoms ranged from 1 hour to 26 days (mean 34.7 \pm 21.1 hours). While 205(68.4\%) presented early, 99 
$(31.6 \%)$ infants presented for surgical care more than 48 hours after the onset of symptoms. There were multiple reasons for presenting late but the most common reason was late referral from the initial hospital of care $28(38 \%)$ (Figure 1).

\section{Diagnosis and surgical procedures}

Anorectal malformation in $69(22.7 \%)$ neonates was the most common diagnosis followed by intestinal (duodenal, jejunoileal, and colonic) atresia in 67 (22.0\%), omphalocele in 27 (8.9\%), gastroschisis in 24 (7.9\%), and esophageal atresia in 23 (7.6\%) neonates. The most common procedures were intestinal resection and anastomosis in 90 (29.6\%), colostomy in $64(21.1 \%)$, primary repair of anterior abdominal wall defect in $39(12.7 \%)$, and silo or modified silo application in 21 (6.9\%) neonates (Table 2). Modified silo implies the use of materials like urine bags, female condoms, and intravenous infusion bags to achieve temporary improvised housing for the intestine. The total number of days on admission was 1 to 81 days $($ Mean $=34.7 \pm 21.1$ days).

One hundred and twenty (39.7\%) neonates had a need for total parenteral nutrition (TPN). However, TPN could not be administered in $74(61.7 \%)$ of them. The reasons were due to non-availability $(29,39.2 \%)$, non-affordability $(5,6.8 \%)$ or both $(40,54.0 \%)$.

\section{Mortality}

There were a total of 81 deaths giving a mortality rate of $26.6 \%$. The fatality rate was highest among those who had a reduction of gastroschisis (15/21, 71.4\%), intestinal resection and anastomoses for atresias, and gut perforations $(34 / 90,37.8 \%)$.

\section{Complications and Determinants of Mortality}

Postoperative Complications: The most common postoperative complications included sepsis (97, $32.0 \%$ ), surgical site infections (88, 28.7\%), malnutrition $(76,25.2 \%)$, hypothermia (59, 19.5\%) and apnea $(51,17.0 \%)$ (Table 3$)$.

Sepsis occurred most frequently in neonates that had intestinal resection and anastomosis (35/90, 38.0\%), colostomy $(17 / 45,37.8 \%)$, and modified silo reduction $(12 / 21,57.1 \%)$. Surgical wound classification type did not correlate with the development of sepsis $(p=0.370)$. Fifty-three $(54.6 \%)$ of the neonates that developed sepsis died $(\mathrm{p}=0.001)$ Again, delayed presentation did not have a statistically significant impact on 30-day mortality $(\mathrm{p}=0.075)$ nor on serious complications like sepsis $(\mathrm{p}=0.131)$.

Of the surgical site infections (SSIs), 66 (21.9\%) were superficial, 15 (5.0\%) were deep and $7(2.3 \%)$ were organ/ space infections. Mortality was 100\% among those that had organ / space infection $(\mathrm{p}=0.001)$, while $8(53.3 \%)$ of those with deep SSIs died $(\mathrm{p}=$ $0.025)$.

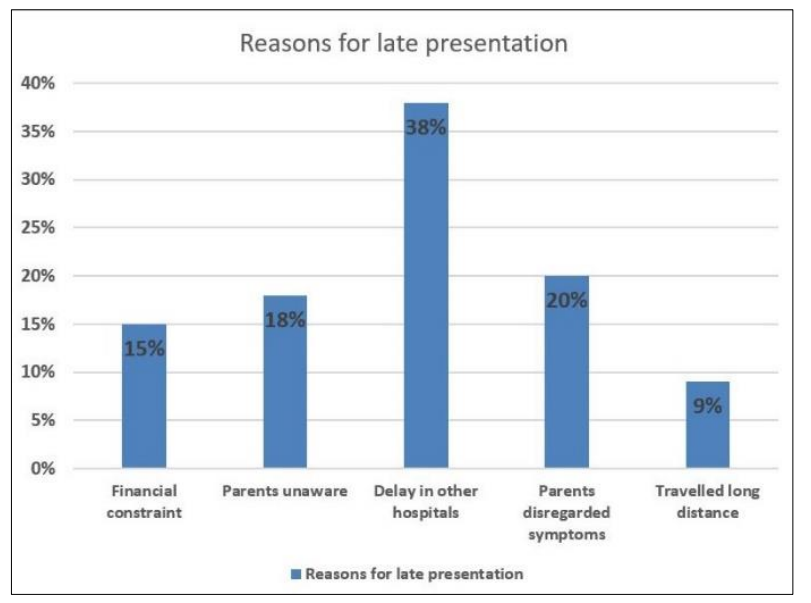

Figure 1: Reasons for late presentation

Table 1: Perinatal characteristics and frequencies

\begin{tabular}{|c|c|c|}
\hline Variable & Frequency & Percentage \\
\hline \multicolumn{3}{|l|}{ Birth-weight (g) } \\
\hline$\leq 1000$ & 2 & 0.7 \\
\hline$>1000 \leq 1500$ & 0 & 0.0 \\
\hline$>1500 \leq 2500$ & 1 & 0.3 \\
\hline$>2500$ & 315 & 99.0 \\
\hline \multicolumn{3}{|l|}{ Gestational age(weeks) } \\
\hline$<28 \quad$ (extremely preterm) & 18 & 5.0 \\
\hline$>28 \leq 32 \quad$ (very preterm) & 11 & 3.6 \\
\hline$>32 \leq 37 \quad$ (late preterm) & 70 & 23.0 \\
\hline$>37 \quad$ (term) & 206 & 67.5 \\
\hline \multicolumn{3}{|l|}{ Mode of delivery } \\
\hline Vaginal delivery & 245 & 80.6 \\
\hline Elective caesarean operation & 12 & 4.0 \\
\hline Emergency caesarean operation & 47 & 15.5 \\
\hline \multicolumn{3}{|l|}{ Place of delivery } \\
\hline Traditional birth attendants(TBA) & 15 & 4.9 \\
\hline Home & 61 & 20.0 \\
\hline Maternity primary health centre & 55 & 18.1 \\
\hline Private hospital & 92 & 30.3 \\
\hline General hospital & 49 & 16.1 \\
\hline Tertiary hospital & 32 & 10.5 \\
\hline \multicolumn{3}{|l|}{$\begin{array}{l}\text { Distance from residence to } \\
\text { hospital }(\mathrm{km})\end{array}$} \\
\hline$<10$ & 34 & 11.2 \\
\hline $11-30$ & 38 & 12.5 \\
\hline $31-50$ & 60 & 19.7 \\
\hline $51-70$ & 52 & 17.1 \\
\hline $71-100$ & 102 & 33.6 \\
\hline$>100$ & 18 & 5.9 \\
\hline \multicolumn{3}{|l|}{$\begin{array}{l}\text { Mode of transportation to the } \\
\text { hospital }\end{array}$} \\
\hline Carried/backed & 25 & 8.2 \\
\hline Motorcycle & 6 & 2.0 \\
\hline Private car & 110 & 36.2 \\
\hline Public transport & 145 & 47.2 \\
\hline Ambulance & 11 & 3.6 \\
\hline Born at the facility & 7 & 2.3 \\
\hline
\end{tabular}


Table 2: showing the common conditions and surgical procedures done with case specific mortalities

\begin{tabular}{|c|c|c|c|c|}
\hline Diagnosis/Surgical Procedures & Frequency & $\%$ & No of mortality & $\begin{array}{l}\text { Disease / procedure specific } \\
\text { mortality rate (\%) }\end{array}$ \\
\hline \multicolumn{5}{|l|}{ Most common disease conditions } \\
\hline Anorectal malformation & 69 & 22.7 & 6 & 8.7 \\
\hline Intestinal atresias & 67 & 22.0 & & \\
\hline Jejunoileal atresia & 35 & 11.5 & 16 & 45.7 \\
\hline Duodenal atresia & 27 & 8.9 & 7 & 25.9 \\
\hline Colonic atresia & 5 & 1.6 & 2 & 40.0 \\
\hline Omphalocoele & 27 & 8.9 & 10 & 37.0 \\
\hline Gastroschisis & 24 & 7.9 & 14 & 58.3 \\
\hline Oesophageal atresia & 23 & 7.6 & 13 & 56.5 \\
\hline Malrotation syndrome & 13 & 4.3 & 2 & 15.4 \\
\hline Necrotising enterocolitis & 11 & 3.6 & 5 & 45.5 \\
\hline Posterior urethral valve & 11 & 3.6 & 0 & 0.0 \\
\hline Gastrointestinal perforation & 4 & 1.3 & 2 & 50.0 \\
\hline Bladder extrophy & 7 & 2.2 & 0 & 0.0 \\
\hline Obstructed inguinal hernia & 4 & 3.4 & 0 & 0.0 \\
\hline Sacrococcygeal tumour & 3 & 1.0 & 0 & 0.0 \\
\hline Infantile hypertrophic pyloric stenosis & 3 & 1.0 & 1 & 33.3 \\
\hline Multiple abscesses & 4 & 1.3 & 2 & 50.0 \\
\hline Hirschsprung's disease & 2 & 0.7 & 0 & 0.0 \\
\hline Omphalopagus twins & 1 & 0.3 & 0 & 0.0 \\
\hline \multicolumn{5}{|l|}{ Most common surgery performed } \\
\hline Intestinal resection/anastomosis & 90 & 29.6 & 34 & 37.8 \\
\hline Colostomy & 64 & 21.1 & 6 & 9.4 \\
\hline Primary closure of abdominal wall defect & 27 & 8.9 & 6 & 22.2 \\
\hline Modified Silo reduction & 21 & 6.9 & 15 & 71.4 \\
\hline Valvotomy & 11 & 3.6 & 0 & 0.0 \\
\hline Feeding gastrostomy & 10 & 3.3 & 7 & 70.0 \\
\hline Anoplasty & 7 & 2.3 & 0 & 0.00 \\
\hline Primary repair of trachea-oesophageal fistula & 8 & 2.6 & 2 & 25.0 \\
\hline Functional bladder closure & 7 & 2.3 & 0 & 0.0 \\
\hline Excision of tumours & 7 & 2.3 & 0 & 0.0 \\
\hline Ladd's procedure & 5 & 2.0 & 1 & 20.0 \\
\hline lleostomy & 5 & 1.7 & 2 & 40.0 \\
\hline Cervical oesophagostomy & 4 & 1.3 & 3 & 75.0 \\
\hline Posterior sagittal anorectoplasty(PSARP) & 4 & 1.3 & 0 & 0.0 \\
\hline Drainage of abscess & 4 & 1.3 & 2 & 50.0 \\
\hline Duodenal web excision & 3 & 1.0 & 1 & 33.3 \\
\hline Pyloromyotomy & 3 & 1.0 & 1 & 33.3 \\
\hline Inguinal herniotomy & 3 & 1.0 & 0 & 0.00 \\
\hline Emergency separation for conjoint twin & 1 & 0.3 & 0 & 0.00 \\
\hline
\end{tabular}


Table 3: Post-operative complications, fatality rates from the complications and correlation with 30-day mortality

\begin{tabular}{|l|c|c|c|c|c|}
\hline Complications & Frequency & Percentage & $\begin{array}{l}\text { No of deaths } \\
\text { attributable to } \\
\text { the complication }\end{array}$ & $\begin{array}{l}\text { Fatality } \\
\text { rate (\%) }\end{array}$ & $\begin{array}{l}\text { p-value (co-relating } \\
\text { complication with 30 } \\
\text { day mortality) }\end{array}$ \\
\hline Sepsis & 97 & 32.0 & 53 & 54.6 & 0.001 \\
\hline Surgical site infection & 88 & 29.2 & 32 & 36.4 & 0.001 \\
\hline Malnutrition & 76 & 25.2 & 57 & 75.0 & 0.001 \\
\hline Hypothermia & 59 & 19.5 & 32 & 54.2 & 0.001 \\
\hline Apnoea & 51 & 17.0 & 37 & 72.5 & 0.001 \\
\hline Renal failure & 27 & 8.9 & 25 & 92.9 & 0.001 \\
\hline Stress gastritis & 22 & 7.3 & 12 & 54.5 & 0.001 \\
\hline Pneumonia & 20 & 6.7 & 17 & 85.0 & 0.001 \\
\hline Prolonged recovery from anaesthesia & 20 & 6.7 & 12 & 60.0 & 0.001 \\
\hline Urinary tract infection & 12 & 4.0 & 1 & 8.3 & 0.139 \\
\hline Unconsciousness & 3 & 1.0 & 3 & 100.0 & 0.003 \\
\hline Seizure & 3 & 1.0 & 2 & 66.7 & 0.116 \\
\hline
\end{tabular}

Catheter-associated urinary tract infection (CAUTI) occurred in $12(5.3 \%)$ of 225 infants that were catheterized; this correlated significantly with the duration of urethral catheterization $(p=0.001)$. The median duration of catheterization was 14 days (interquartile range (IQR) 1 - 26 days). The risk of development of UTI from catheterization increased by 1.05 times after 72 hours (OR 1.05, $95 \% \mathrm{CI}=0.28$ 3.98 ) and 3.9 times after 7 days (OR 3,88, 95\%CI= $0.31-47.17)$ on the urethral catheter. More boys (9) had UTI than girls $(3)(\mathrm{p}=0.39)$.

Apnea and Anesthesia: Postoperative apnea, the most frequent anesthetic complication occurred in $51(17.0 \%)$ neonates. Thirty-six neonates died from anesthesia-related complications representing $44.4 \%$ of the total mortality. The risk of mortality increased by 11 times in those that developed apnea $(\mathrm{OR}=10.83, \mathrm{p}=0.001,95 \% \mathrm{CI}=5.47-21.44)$.

Anesthesia time lasted more than 2 hours in $198(66.0 \%)$ neonates. Anesthesia time longer than 2 hours did not correlate with the development of apnea $(\mathrm{p}=0.67)$ and 30-day mortality $(\mathrm{p}=0.50)$.

Mortality was higher with the high ASA class. Mortality among ASA I was $21.9 \%(n=25 / 114)$, and ASA II was $20.3 \%(n=16 / 79$,$) ; while ASA III was 36.5 \%$ $(n=31 / 85)$, ASA IV was $38.1 \%(n=8 / 21)$ and ASA V was $20 \%(\mathrm{n}=1 / 5)$. P-value was insignificant at $=0.07$.

Residents in anesthesiology administered anesthesia in $179(60,0 \%)$ of the cases, while consultants did in $92(30.8 \%)$ and anesthetic nurses $27(9.1 \%)$. Mortality across the groups was not significant $(p=0.814)$.

Distance from Hospital: Although 172 (56.96\%) neonates traveled more than $50 \mathrm{~km}$ to access care, there was no associated significant risk of death compared to those closer to the hospital $(\mathrm{OR}=0.83,95 \% \mathrm{CI}$ 0.50-1.39, $\mathrm{p}=0.48)$.

Gestational Age and Birth Weight: While 99 (32.5\%) patients were preterm, only $3(0.9 \%)$ neonates weighed less than 2500 gm at birth. Proportionately, more deaths occurred in the preterm (39/99, fatality rate $39.4 \%)$ compared to the term neonates $(42 / 205$, fatality rate $20.5 \%)(p=0.001)$.

Other factors: Consultant pediatric surgeons performed $196(64.5 \%)$ of the initial surgical procedures while Senior Registrars (trainees in pediatric surgery) did $87(28.6 \%)$ and Registrars (junior surgical trainees) did $21(6.9 \%)$. Mortality did not differ significantly across groups $(\mathrm{p}=0.760)$.

Re-operation increased the risk of mortality by 2.9 times (OR 2.93, 95\% CI I.06-8.09, p=0.038). Sixteen $(5.7 \%)$ neonates were re-operated; 9 (3.0\%) were operated twice and $7(2.7 \%)$ thrice. Repeat operations were done within 30 days of the first. Indications for reoperation were failed intestinal anastomosis for intestinal atresia (9), complications of anterior abdominal wall defect closure (3), colostomy revision (1), gastrostomy revision (1), failed gastric perforation closure (1), and complications from sacrococcygeal tumor excision (1).

Oxygen saturation was measured and recorded at admission in only $81(26.6 \%)$ neonates. Out of these, $40(23.8 \%)$ neonates were admitted with oxygen saturation of less than $90 \%$. $\mathrm{SpO} 2$ at admission less than $90 \%$ did not adversely affect the 30-day mortality $(p=0.20)$. Thirty-one neonates had postoperative cardiac arrest, 29(93.6\%) of them died $(p=0.001)$. Another two died intra-operation. 


\section{Risk Predictors}

Logistic regression analysis showed that the risk of death is highest in those that developed apnea $(\mathrm{OR}=10.8)$. Other risk predictors are shown in Table 4.

Table 4: Determinants of mortality (binary logistic regression analysis)

\begin{tabular}{|l|l|l|l|l|}
\hline Variable & $\begin{array}{l}\text { Odds } \\
\text { Ratio }\end{array}$ & $\begin{array}{l}\text { Std } \\
\text { Error(b) }\end{array}$ & $\begin{array}{l}\boldsymbol{P} \mathbf{[}[\mathbf{z}] \\
\mathbf{9 5 \%} \\
\text { OR }\end{array}$ \\
\hline Apnoea & 10.83 & 3.78 & 0.001 & $5.47-21.44$ \\
\hline Sepsis & 7.61 & 2.19 & 0.001 & $4.33-13.39$ \\
\hline Malnutrition & 6.99 & 2.05 & 0.001 & $3.93-12.43$ \\
\hline Deep SSI & 3.50 & 1.88 & 0.025 & $1.22-10.01$ \\
\hline Re-operation & 2.93 & 1.52 & 0.038 & $1.06-8.09$ \\
\hline CI-confidence interval OR-Odds ratio & SSI-surgical site infection
\end{tabular}

\section{DISCUSSION}

\section{Mortality}

A systematic review comparing mortality in surgical neonates in Africa in the last two decades showed an improvement from a rate of $36.9 \%$ to $29.1 \%$. [11] The overall mortality rate of neonatal surgery in Nigeria in this study was $26.6 \%$. Although this compares with the overall trend reported across Africa, it is still more than double the projected SDG 3.2 target and much higher when compared to less than $5 \%$ reported in high-income countries. [15]

Comparing different systems, gastrointestinal pathologies contributed to the overwhelming majority $(76$, $93.8 \%$ ) of the mortalities in the present report of general pediatric surgical neonates. Most mortalities were from surgery for intestinal atresias, gastroschisis, esophageal atresia, omphalocele, anorectal malformation, and intestinal malrotation, similar to previous single institutional reports from Nigeria and Africa, $[16,17]$ but much higher than HICs. For instance, the mortality from gastroschisis in the present report was $58.3 \%$ compared to $1 \%$ in HICs. [18] Most of the patients with gastroschisis were managed using an improvised surgical silo (made from several materials, including urine bag, female condom, intravenous fluid bag, and surgical gloves). Incidentally, most of the deaths were from sepsis and aspiration pneumonitis. The $70 \%$ mortality from this method of treatment may be explained by the absence of a protocol to improve outcomes. Wesonga et al were able to reduce gastroschisis mortality in Uganda from $90 \%$ to $58 \%$ in one year by using a locally designed protocol. [19]

The absence of NICU and support facilities like in most sub-Saharan Africa no doubt also played a role in the high mortality. The majority of the neonates were managed in special care baby and high dependency units. Many other improvisations were often made as regards temperature control and nutritional needs of the neonates. Patient monitoring also often takes a lot of man-hours from the surgical teams due to the paucity of intensivists and monitoring gadgets.

\section{Morbidities}

As shown in Table 3, the occurrence of postoperative complications was common and greatly affected the survival of the neonates. Sepsis has been documented in several reports from developing countries as the most common cause of neonatal postoperative death. [9, 20, 21] Neonates have deficient cellular and humoral immunity; surgery additionally breaches the physical barrier to organisms, further increasing the risk of SSI and sepsis. From our study, nearly onethird of the neonates had sepsis, and more than half of this number died. Delayed presentation unlike in many LMICs did not significantly affect the development of sepsis in our study $(p=0.075)$. As observed from the study, the major reason for the delayed presentation was a late referral from peripheral hospitals (32.8\%). However, most of these neonates must have been on antibiotics asides from other resuscitative measures prior to arrival at the specialist centers, thus accounting for the low incidence of sepsis among late presenters. On the other hand, it might be possible that poor unsupervised deliveries at home, long and unsupervised transportation as well as nursing surgical neonates in sick pediatric wards as seen in many LMICs may have contributed to the overall high rates of SSI and sepsis. [22] Surgical site infection is common in sub-Saharan Africa and significantly affects the postoperative outcome. [7, 23]

Besides pre-existing preoperative malnutrition, a catabolic metabolic response from disease, surgery, and delayed onset of feeding also pushes the neonate further into negative nutritional balance, increasing the risk of death. Over $60 \%$ of infants that needed TPN never received it due to non-availability and nonaccessibility, a situation that also exists in most subSaharan African countries. [7] Further studies on the burden of neonatal surgical malnutrition, the development of surgical nutrition guidelines, and the provision of TPN are needed to improve outcomes.

\section{Challenges of anesthesia}

In the present report, anesthesia-related deaths accounted for $44.4 \%$ of overall mortality, with the development of postoperative apnea as the most common risk factor. The most used modality was general anesthesia indicating an improvement in the choice of anesthesia for neonatal surgeries over the earlier preferred choice of local anesthesia. [24] This may be explained by an increase in the number of physician anesthetists involved in neonatal surgeries. In one report from Ghana, an increase in the number of physician anesthetists contributed to improving the outcome of neonatal surgery. [25] However specialist pediatric anesthetists are still quite a few, and the 
density of anesthetists in Nigeria is still low. [26] Encouraging and training pediatric anesthetists along with the provision of appropriate facilities should lead to improvements in the safety and outcome of anesthesia in the setting.

\section{Preoperative factors}

Majority of the neonates traveled more than $50 \mathrm{~km}$ to the hospital of care on public transportation unsupervised. While $20 \%$ were born at home with poor care, only a small fraction $(2.3 \%)$ was born in the tertiary hospital of care. The low in-hospital delivery might be related to low prenatal diagnosis. Low utilization and uptake of prenatal ultrasonography especially in rural areas of sub-Saharan Africa where access to ultrasound services may be as low as $6 \%$ has been documented. [11, 27] The long-distance travel and low in-hospital delivery may worsen the physiologic status of the already severely ill infants, and some may die before accessing care. [24]

Again, it is noteworthy that the majority of the reported cases in this study weighed $\geq 2.5 \mathrm{~kg}$. The preterm and low birth weight neonates are probably not reaching the pediatric surgeons and are a subject for further research.

At present, most pediatric surgeons in Nigeria are still concentrated mainly in the tertiary institutions in the urban areas leaving a vast population in rural areas without access to a pediatric surgeon. [28] The majority of the infants were referred from these distant rural areas.

\section{Conclusion}

Although there have been some improvements in the practice of neonatal surgery in Nigeria, the national mortality is still high at $26.6 \%$. The outcome is worse in the preterm and in neonates that developed apnea, sepsis, or disease-induced malnutrition.

\section{Recommendation}

Development and implementation of treatment protocols and guidelines; and collaboration across centers is needed to ensure a continuous neonatal surgical quality improvement for a sustained good outcome. District and secondary level hospitals should be equipped and staffed to handle basic

\section{REFERENCES}

1. World Health Organisation. SDG 3: Ensure healthy lives and promote wellbeing for all at all ages. Available at www.who.int/sdg/targets/en/. Accessed June 24, 2020.

2. UNICEF. Child survival and the SDGs. Available at data.unicef.org/topic/child-survival/child-survivalsdgs/. Accessed June 24, 2020.

3. Oza S, Lawn JE, Hogan DR, Mathers C, Cousens SN. Neonatal cause-of-death estimates for the early and late neonatal surgical emergencies along with strengthening of referral and transfer strategies to higher-level hospitals. Advocacy should be increased towards the training of more pediatric surgeons, pediatric anesthetists, and support staff and motivating them to work in the district and secondary level hospitals.

\section{Acknowledgements: Nil}

Conflict of Interest: None decalred

\section{Source of Support: Nil}

Consent to Publication: Author(s) declared taking informed written consent for the publication of clinical photographs/material (if any used), from the legal guardian of the patient with an understanding that every effort will be made to conceal the identity of the patient, however it cannot be guaranteed.

Author Contributions: Author(s) declared to fulfil authorship criteria as devised by ICMJE and approved the final version.

\section{Authors' Affiliation}

1.Surgery Department, Nnamdi Azikiwe University / Nnamdi Azikiwe University Teaching Hospital Nnewi, Anambra State Nigeria.

2. Department of Surgery, National Hospital Abuja, Nigeria

3. Division of Paediatric Surgery, Department of Surgery, University of Ilorin / University of Ilorin Teaching Hospital Ilorin, Nigeria

4. Pediatric Surgery Unit, Department of Surgery, Faculty of Clinical Sciences, College of Medicine, University of Lagos Idi Araba PMB 12003 Lagos, Nigeria

5. Surgery Department, University of Uyo Teaching Hospital Uyo, Nigeria

6. Department of Surgery, Abubakar Tafawa Balewa University Teaching Hospital Bauchi

7. Division of Paediatric Surgery, Department of Surgery, University College Hospital Ibadan, Nigeria

8. Sub-Department of Paediatric Surgery, University of Nigeria Teaching Hospital Ituku-Ozalla Enugu, Nigeria

9. Department of Surgery, Lagos State University Teaching Hospital Ikeja Lagos, Nigeria

10. Department of Surgery, Usmanu Danfodiyo University Teaching Hospital Sokoto, Nigeria

11. Paediatric Surgery Division, Department of Surgery, OlabisiOnabanjo University Teaching Hospital Sagamu, Ogun State Nigeria

12. Department of Surgery, Alex Ekwueme Federal University Teaching Hospital Abakaliki, Nigeria

13. Pediatric Surgery Unit, Department of Surgery, University of Port Harcourt/ University of Port Harcourt Teaching Hospital, Port Harcourt, Rivers State Nigeria

14. Division of Paediatric Surgery, Department of Paediatric Surgery, University of Abuja Teaching Hospital Gwagwalada, Nigeria

15. Division of Paediatric Surgery, Ahmadu Bello University Teaching Hospital Zaria, Nigeria

16. Department of Surgery, Obafemi Awolowo University Teaching Hospital, Ile-Ife Osun State, Nigeria

17. Department of Surgery, Federal Medical Centre, Lokoja, Kogi State

neonatal periods for 194 countries: 2000-2013.Bulletin of the World Health Organization 2015; 93:19-28. Available https: / /www.who.int/bulletin/volumes/93/1/BLT-14139790-table-T2.html.

4. Woods D, Aldous C, Christianson A, Malherbe HL. The contribution of congenital disorders to child mortality in South Africa. South Afr Heal Rev. 2016; 1:137-52. 
5. UNICEF. Neonatal mortality rate. UNICEF global database. Available at https://data.unicef.org/wpcontent/uploads/2017/10/Neonatal-mortalityrate_2020.xlsx. Accessed September 27, 2020.

6. Lohfa B Chirdan, Petronila JN, Essam A Elhalaby. Neonatal surgery in Africa. Semin Pediatr Surg. 2012; 21:151-9.

7. Ameh EA, Seyi-Olajide JO, Sholadoye TT. Neonatal surgical care: a review of the burden, progress and challenges in sub-Saharan Africa. Paediatr Int Child Heal. 2015; 35:243-51.

8. Ilori IU, Ituen AM, Eyo CS. Factors associated with mortality in neonatal surgical emergencies in a developing tertiary hospital in Nigeria. Open $\mathrm{J}$ Paediatr. 2013; 3:35890. Available from https://doi.org/10.4236/ojped.2013.33040.

9. Ekwunife $\mathrm{OH}$, Okpata A, Ugwu JO, Osuigwe AN. Outcome of neonatal surgeries in Nnewi, Nigeria. Ann Pediatr Surg. 2015; 11:132-5.

10. Ugwu RO, Okoro PE. Pattern, outcome and challenges of neonatal surgical cases in a tertiary teaching hospital. Afr J Paediatr Surg. 2013; 10:226-30.

11. Ekenze SO, Ajuziogu OV, Nwomeh BC. Neonatal surgery in Africa: a systematic review and metaanalysis of challenges of management and outcome. Lancet. 2015; 385:S35.

12. American College of Surgeons. Paediatric ACS NSQIP operations manual 2015. Available from https://www.google.com/url?sa=t\&source=web\&rct=j\& url=https://www.facs.org/ /media/files / quality\%2520 programs/nsqip/nsqip_puf_user_guide_2015.ashx\&ved $=2 \mathrm{ahUKEwj} 5 \mathrm{iKrgo5n} 1 \mathrm{AhVNzBoKHb4QA74QFnoECAYQ}$ AQ\&usg=AOvVaw25Y_eUNTPGjYy9iQiq5Cqo

13. Aiwanlehi E, Ogbaisi E. Pattern of neonatal surgery at a teaching hospital in Nigeria: A review of 101 cases. East Cen Afr J Surg. 2013; 18:56-60.

14. Sample size calculator. Available from: www.surveysystem.com/sscalc.html.

15. Yagi M, Kohno M, Asagiri K, Ikeda T, Okada T, Kanada $\mathrm{S}$, et al. Twenty-year trend in neonatal surgery based on a nationwide Japanese surveillance program. Pediatr Surg Int. 2015; 31:955-62.

16. Livingstone $\mathrm{MH}$, Cruz J, Pemberton J, Ozgediz $Z$, Poenaru D. Mortality of pediatric surgical conditions in low and middle income countries. J Pediatr Surg. 2015; 50:760-4.

17. Nandi B, Mungongo C, Lakhoo A. A comparison of neonatal surgical admissions between two linked surgical departments in Africa and Europe. Pediatr Surg Int. 2008; 24 :939-42.

18. Manson J, Ameh E, Canvassar N, Chen T, Hoeve A, Lever F, et al. Gastroschisis: A multi-centre comparison of management and outcome. Afr $\mathrm{J}$ Paediatr Surg. 2012; 9:17-21.

19. Wesonga A, Situma M, Lakhoo K. Reducing gastroschisis mortality: A quality improvement initiative at a Ugandan Paediatric Surgery Unit. World J Surg. 2020; 44:1395-9. Available from: https://doi.org/10.1007/s00268-020-05373-w.

20. Hassan MS, Rahman A, Huq U. Neonatal surgical mortalities in developing countries: Can it be a barrier to achieving sustainable development goals (SDG). Int $\mathrm{J}$ Pediatr Child Care. 2019; 1:1-4.

21. Ademuyiwa AO, Sowande OA, Ijaduola TK, Adejuyigbe O. Determinants of mortality in neonatal intestinal obstruction in Ile Ife, Nigeria. Afr J Paediatr Surg. 2009; 6:11-3.

22. Mitul AR. Surgical neonatal sepsis in developing countries. J Neonatal Surg. 2015; 4:41.

23. Ekenze SO, Ajuzieogu OV, Nwomeh BC. Challenges of management and outcome of neonatal surgery in Africa: A systematic review. Pediatr Surg Int. 2016; 32:291-9. Available from: https://doi.org/10.1007/s00383-0163861-x.

24. Ameh EA, Ameh N. Providing safe surgery for neonates in Sub-Saharan Africa. Trop Doct. 2003; 33:145-7.

25. Amponsah G. Challenges of anaesthesia in the management of surgical neonates in Africa. Afr $\mathrm{J}$ Paediatr Surg. 2010; 7:134-9.

26. National Surgical, Obstetrics, Anaesthesia \& Nursing Plan (NSOANP) for Nigeria: Strategic Priorities for Surgical Care (StraPS) (2019-2023) [Internet]. Federal Ministry of Health, Nigeria; 2019 [cited 2020 Aug 17]. Available from: http://health.gov.ng/doc/NSOANP.pdf.

27. Akinmoladun JA, Anumba DO. Fetal imaging and diagnosis services in developing country- A call to action. Trop J Obstet Gynaecol. 2019; 36:11-7.

28. Ameh EA, Adejuyigbe O, Nmadu PT. Paediatric surgery in Nigeria. J Pediatr Surg. 2006; 41:542-6. 